\title{
Change of Fecal Flora and Effectiveness of the Short-term VSL\#3 Probiotic Treatment in Patients With Functional Constipation
}

\author{
Seong-Eun Kim, ${ }^{1}$ Suck Chei Choi, ${ }^{2 *}$ Kyung Sik Park, ${ }^{3}$ Moo In Park, ${ }^{4}$ Jeong Eun Shin, ${ }^{5}$ Tae Hee Lee, ${ }^{6}$ Kee Wook Jung, ${ }^{7}$ Hoon \\ Sup Koo, ${ }^{8}$ Seung-Jae Myung ${ }^{7}$, and Constipation Research group of Korean Society of Neurogastroenterology and Motility \\ ${ }^{1}$ Ewha Womans University School of Medicine, Seoul, Korea; ${ }^{2}$ Wonkwang University School of Medicine, Iksan, Jeollabuk-do, Korea; \\ ${ }^{3}$ Keimyung University School of Medicine, Daegu, Korea; ${ }^{4}$ Kosin University College of Medicine, Busan, Korea; ${ }^{5}$ Dankook University College \\ of Medicine, Cheonan, Chungcheongnam-do, Korea; ${ }^{6}$ Soonchunhyang University College of Medicine, Seoul, Korea; ' University of Ulsan \\ College of Medicine, Seoul, Korea; and ${ }^{8}$ Konyang University College of Medicine, Daejon, Korea
}

\begin{abstract}
Background/Aims
We investigated gut flora characteristics in patients with functional constipation (FC) and influences of short-term treatment with VSL\#3 probiotic on flora and symptom improvement.

\section{Methods}

Thirty patients fulfilling Rome III criteria for FC and 30 controls were enrolled. Fecal samples were obtained before and after VSL\#3 intake (one sachet twice daily for 2 weeks) and flora were examined by quantitative real-time polymerase chain reaction (qRT-PCR). Symptom changes were also investigated.
\end{abstract}

\section{Results}

The fold differences in Bifidobacterium and Bacteroides species were significantly lower in feces from FC, compared to in controls $(P=0.030$ and $P=0.021)$. After taking VSL\#3, the fold differences in Lactobacillus, Bifidobacterium and Bacteroides species increased in controls $(P=0.022, P=0.018$, and $P=0.076)$, but not in FC. Mean Bristol scores and complete spontaneous bowel movements (CSBMs)/week increased significantly in FC after ingesting VSL\#3 (both $P<0.001$ ). Relief of subjective CSBM frequency, stool consistency and abdominal bloating were reported in $70 \%, 60 \%$, and $47 \%$ of patients. After VSL\#3 cessation, $44.4 \%$ of patients with symptom improvement experienced constipation recurrence mostly within one month.

\section{Conclusions}

Bifidobacterium and Bacteroides species might be quantitatively altered in FC. A short-term VSL\#3 treatment can improve clinical symptoms of FC. Further studies are needed to investigate VSL\#3's additional effects beyond altering gut flora to allevate constipation.

(J Neurogastroenterol Motil 2015;21:111-120)

\section{Key Words}

Constipation; Microbiome; Probiotics

Received: April 14, 2014 Revised: August 20, 2014 Accepted: September 1, 2014

(c) This is an Open Access article distributed under the terms of the Creative Commons Attribution Non-Commercial License (http://creativecommons. org/licenses/by-nc/3.0) which permits unrestricted non-commercial use, distribution, and reproduction in any medium, provided the original work is properly cited.

*Correspondence: Suck Chei Choi, MD, PhD

Department of Internal Medicine and Digestive Disease Research Institute, Wonkwang University College of Medicine, 460 Iksan-daero, Iksan, Jeollabuk-do 570-974, Korea

Tel: +82-63-859-2563, Fax: +82-63-855-2025, E-mail: medcsc@wmc.wonkwang.ac.kr

Financial support: This study was supported by NAMU Co, Korea; and a Daewoong research grant from the Korean Society of Neurogastroenterology and Motility.

Conflicts of interest: None.

Author contributions: Seong-Eun Kim, Suck Chei Choi, and Moo In Park designed the research study; Seong-Eun Kim performed the research (analysis of gut flora); Seong-Eun Kim, Suck Chei Choi, and Seung-Jae Myung wrote the paper; Kee Wook Jung, Hoon Sup Koo, and Seong-Eun Kim analyzed the data; Kyung Sik Park, Moo In Park, Jeong Eun Shin, Tae Hee Lee, and Seong-Eun Kim collected human samples.

ORCID: Kyung Sik Park, http://orcid.org/0000-0003-1874-9936; Tae Hee Lee, http://orcid.org/0000-0003-3049-8252. 


\section{Introduction}

The gut flora is a collection of microorganisms that live within the intestine, creating a harmonious ecosystem with concentrations of up to $10^{11}-10^{12}$ cells/g luminal contents and which represent approximately $60 \%$ of the fecal mass. ${ }^{1}$ The gut flora performs many important health-promoting functions such as metabolic activities, fermenting unused energy substrates, producing vitamins or hormones to direct the host to store fats, immune system development, and preventing growth of pathogenic bacteria. ${ }^{1-3}$ Moreover, recent studies have shown that gut flora may play a role in gut sensory and motor functions. ${ }^{4,5}$

Although a change in gut flora has been suggested as a possible pathogenesis of functional bowel disease, few studies exist on the relationship between functional constipation (FC) and gut flora. In a pediatric study, constipated children presented with a significant increase in Clostridia and Bifidobacteria in feces compared to healthy subjects. ${ }^{6,7}$ Another study showed that concentrations of Bifidobacterium and Lactobacillus were significantly lower in constipated patients. ${ }^{8}$ These previous studies evaluated standard microbial cultures using selective media, but the methods lacked reproducibility. Approximately $40-80 \%$ of bacteria observed by a direct microscopic examination are not recoverable by culture, although estimates vary among individuals and studies . Additionally, the culture method can not detect both dead and live microbes in fecal samples, which is another limitation. Advances in bacterial taxonomy based on molecular methods, especially comparison of the $16 \mathrm{~S}$ ribosomal RNA gene, have made cultivation-independent investigation the standard approach for microbial community analysis. ${ }^{10,11}$

The influence of gut flora on FC can be assessed by examining the symptomatic effects of probiotics administered to FC patients. Several studies have suggested probiotics as a possible treatment agent. ${ }^{12-14}$ The therapeutic outcome of a specific probiotic in the management of patients with $\mathrm{FC}$ depends on various factors, including bacterial strains, treatment duration, administration form and dose, and host factors. Currently, due to the paucity of data, whether any particular probiotic is more effective in the treatment of FC remains inconclusive. Additionally, whether the improvement of symptoms is directly due to a change in gut flora itself is unclear.

Therefore, in this study we investigated the characteristics of gut microbiome in patients with FC using molecular methods and evaluated the influence of a short-term treatment with
VSL\#3 probiotic, consisting of multiple viable lactic acid bacteria, on gut flora and the improvement of symptom profiles. Similar studies have not been reported previously, thus we performed this pilot study in preparation for larger and more advanced studies.

\section{Materials and Methods}

\section{Eligibility}

We recruited $30 \mathrm{FC}$ patients and 30 healthy controls from 6 university hospitals between October 2011 and August 2012. The inclusion criteria were as follows: 20-59 years of age; fulfilling Rome III criteria for FC for at least 5 years; availability of at least one gastrointestinal (GI) imaging study during the last 5 years; and availability for the entire study period. Healthy controls were enrolled after excluding of any GI complaints and colonoscopies were performed in controls over 40 years of age to eliminate other organic bowel diseases.

Subjects with other GI symptoms, a history of GI surgery, organic intestinal diseases, lactose malabsorption, pregnant or lactating, or severe systemic diseases including diabetes, cardiovascular problems, or neurologic diseases were excluded. Subjects with a history of antibiotic treatment or intentional probiotic consumption 1 month prior to starting this study were also excluded.

All participants provided written informed consent according to institutional guidelines, and the protocol was approved by the Institutional Review Board of the participating hospitals.

This study was registered at the Clinical Research Information Service (CRiS), registration number KCT0000448.

\section{Study Design}

This was a non-randomized controlled study evaluating the characteristics of fecal flora in FC and the comparative effects of probiotics between the $\mathrm{FC}$ and control groups. In the protocol, a 1 -week run-in observation period was followed by a 2 -week treatment period, with fecal samples obtained before and after the treatment period. All subjects received a VSL\#3 sachet (VSL Pharmaceuticals, Danisco, USA) twice daily for 2 weeks. Each VSL\#3 sachet contained 450 billion lyophilized bacteria: Bifidobacterium (B. longum, B. infantis and B. breve); Lactobacillus ( $L$. acidophilus, L. casei, L. bulgaricus, and L. plantarum); and Streptococcus thermophilus.

During the study period, subjects were required to record a 
daily diary of bowel function including complete spontaneous bowel movements (CSBMs) and stool consistency using a validated Bristol stool scale, ${ }^{15}$ as well as respond weekly to the following questions on the symptomatic relief of FC: "During the past seven days, have you experienced satisfactory relief of bowel movement frequency, stool consistency, or bloating?" Diet and drugs taken during the trial were recorded. Patients with FC were not strongly encouraged to quit their usual laxatives.

Subjects who showed symptomatic improvement of functional constipation were followed up monthly by telephone interview for 6 months.

Stool samples collected from all subjects were stored at $-80^{\circ} \mathrm{C}$ for DNA extraction and quantitative real-time polymerase chain reaction (qRT-PCR).

\section{Fecal Microbiological Analyses}

Fecal DNA was extracted using the QIAamp stool DNA Extraction kits (Qiagen, Valencia, CA, USA) according to manufacturer's instructions. Quantitative RT-PCR was performed using the ABI SYBR Green PCR Mastermix from Applied Biosystems (Foster City, CA, USA), with primers amplifying the genes encoding $16 \mathrm{~S}$ rRNA from specific bacterial groups including Bacteroides spp., Clostridium spp., Escherichia coli, Bifidobacterium spp., and Lactobacillus spp.(Table 1). ${ }^{16}$ Quantitative RT-PCR assays were conducted in 96-well plates using a ABI PRISM7000 system (Applied Biosystems). Each qRT-PCR was performed duplicately in a final volume of $20 \mu$ l containing $1 \times$ SYBR Mastermix, $0.5 \mu \mathrm{M}$ of each primer and $50 \mathrm{ng}$ of purified fecal DNA. Quantitative RT-PCR conditions were as follows: $15 \mathrm{mi}-$ nutes at $95^{\circ} \mathrm{C}$, followed by 40 cycles of $95^{\circ} \mathrm{C}$ for 1 minute, $30 \mathrm{sec}-$ onds at the appropriate annealing temperature, and $72^{\circ} \mathrm{C}$ for 1 minute. Quantitative RT-PCR standards were generated by PCR amplifying and cloning the target $16 \mathrm{~S}$ rRNA from an ap- propriate positive control strain. Melting curve analysis of qRT-PCR products was conducted following each assay to confirm the fluorescence signal originated from specific products and not from primer-dimers or artifacts. All qRT-PCR plates included a 'no template' negative control for each primer set. All microbiology analyses of fecal samples were performed in a blinded manner, without knowledge of the subjects' information. The relative gene expression of gut flora was normalized to $16 \mathrm{~S}$ rRNA expression, the internal reference, in fecal samples using the comparative $\mathrm{C}_{\mathrm{T}}$ (threshold cycle) method. ${ }^{17}$ The concentrations of each bacteria in constipated patients or treated group were expressed as a quantitative "fold difference" of bacterial $\Delta \mathrm{Ct}$ values in the subjects compared to mean $\Delta \mathrm{Ct}$ values of control group or pretreated group using the formula of exponential $2^{-\Delta \Delta \mathrm{C}_{\mathrm{T}}}$ :

$$
2^{-\Delta \Delta \mathrm{C}_{\mathrm{T}}}=2^{-\left[\left(\mathrm{C}_{\mathrm{T}} \text { gene of specific bacterial species }-\mathrm{C}_{\mathrm{T}} 16 \mathrm{~S} \mathrm{rRNA} \text { per } \mu \mathrm{g} \text { of sample }\right)\right.}
$$

\section{Statistical Methods}

This study included 30 patients with FC and 30 controls. Continuous variables are reported as medians with range and/or means $\pm \mathrm{SD}$, and categorical variables as relative frequencies.

The CSBM scores and Bristol scales are summarized as the means \pm SD before and after VSL\#3 ingestion. The comparative analysis of symptomatic scores was performed by paired $t$ test.

Fold differences of 5 bacterial species before and after probiotic treatment were compared using the Wilcoxon signed-rank test. Fold differences in the bacterial species between FC patients and controls were compared using the Mann-Whitney test. All $P$-values were two-sided and $P$-values $<0.05$ were considered to indicate significance. All analyses were performed using SPSS software, version 18.0 (SPSS Inc., Chicago, IL, USA).

Table 1. Quantitative Polymerase Chain Reaction Primers

\begin{tabular}{|c|c|c|c|c|}
\hline Target bacteria & & Primer sequence $5^{\prime}-3^{\prime}$ & Annealing temperature $\left({ }^{\circ} \mathrm{C}\right)$ & PCR product size (base pair) \\
\hline \multirow[t]{2}{*}{ Bacteroides spp. } & F- & ATAGCCTTTCGAAAGRAAGAT & 50 & 495 \\
\hline & R- & CCAGTATCAACTGCAATTTTA & & \\
\hline \multirow{2}{*}{ Clostridium spp. } & F- & CGGTACCTGACTAAGAAGC & 50 & 429 \\
\hline & R- & AGTTTYATTCTTGCGAACG & & \\
\hline \multirow[t]{2}{*}{ Escherichia coli } & F- & GTTAATACCTTTGCTCATTGA & 52 & 340 \\
\hline & & ACCAGGGTATCTAATCCTGTT & & \\
\hline \multirow[t]{2}{*}{ Bifidobacterium spp. } & F- & GGGTGGTAATGCCGGATG & 55 & 442 \\
\hline & R- & TAAGCGATGGACTTTCACACC & & \\
\hline \multirow[t]{2}{*}{ Lactobacillus spp. } & F- & AGCAGTAGGGAATCTTCCA & 50 & 341 \\
\hline & R- & CACCGCTACACATGGAG & & \\
\hline
\end{tabular}




\section{Results}

\section{Subjects' Clinical Characteristics}

A total of 60 subjects ( $30 \mathrm{FC}$ and 30 controls) provided fecal samples. Females represented $65 \%$ of the subjects, with a mean age of 33 years (range 22-42 years). The mean age of patients

Table 2. Clinical Characteristics of Subjects

\begin{tabular}{lcc}
\hline & FC patients & Healthy controls \\
\hline Number of subjects & 30 & 30 \\
Age $($ mean $\pm \mathrm{SD}, \mathrm{yr})$ & $35 \pm 5^{\mathrm{a}}$ & $32 \pm 3$ \\
Male/female & $9 / 21$ & $12 / 18$ \\
BMI $\left(\right.$ mean $\left.\pm \mathrm{SD}, \mathrm{kg} / \mathrm{m}^{2}\right)$ & $21.0 \pm 2.5$ & $21.5 \pm 2.9$
\end{tabular}

$\mathrm{FC}$, functional constipation. ${ }^{\mathrm{a}} P=0.010$ vs. healthy controls.

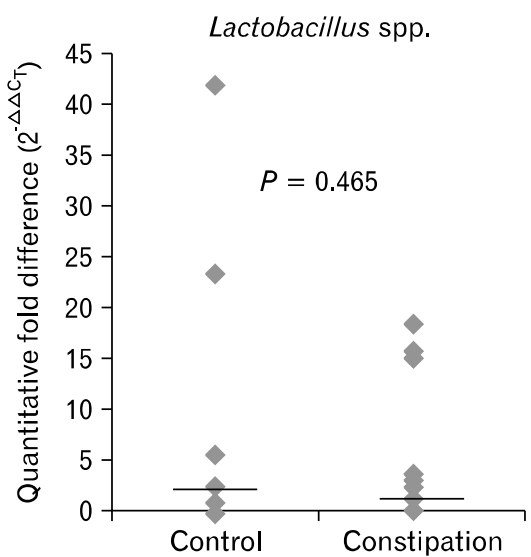

with $\mathrm{FC}$ was significantly greater than that of controls ( $35 \pm 5$ vs. $32 \pm 3$ years, respectively; $P=0.010)$. Body mass index (BMI) was similar in the 2 groups (Table 2). Most patients with FC did not take a laxative during the study period, and only 2 patients used bisacodyl tablets and suppositories intermittently as rescue treatments when they did not have a bowel movement for 4 days. All subjects took VSL\#3 for 2 weeks.

\section{Fecal Microbiology by Quantitative Real-time Polymerase Chacin Reaction}

\section{Comparison of fold differences in concentrations of gut flora between functional constipation patients and controls}

Fecal qRT-PCR showed a significant low value in Bifidobacterium and Bacteroides species in fecal specimens from constipated patients when compared to healthy controls $(P=0.030$ and $P=$ $0.021)$. No significant differences were observed in the fold dif-

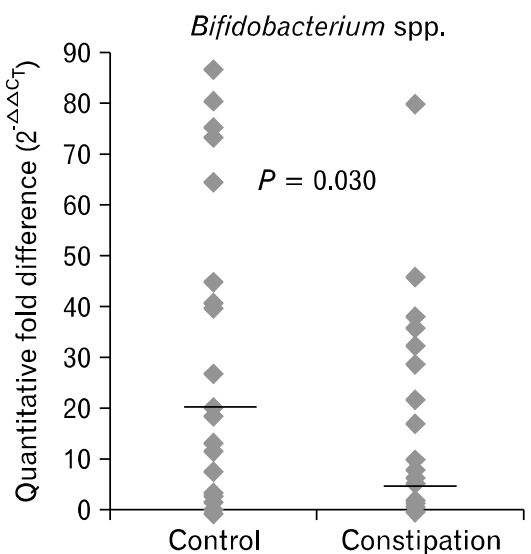

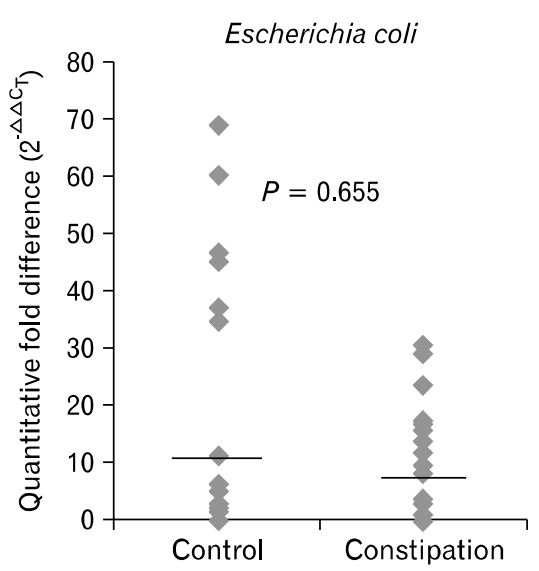
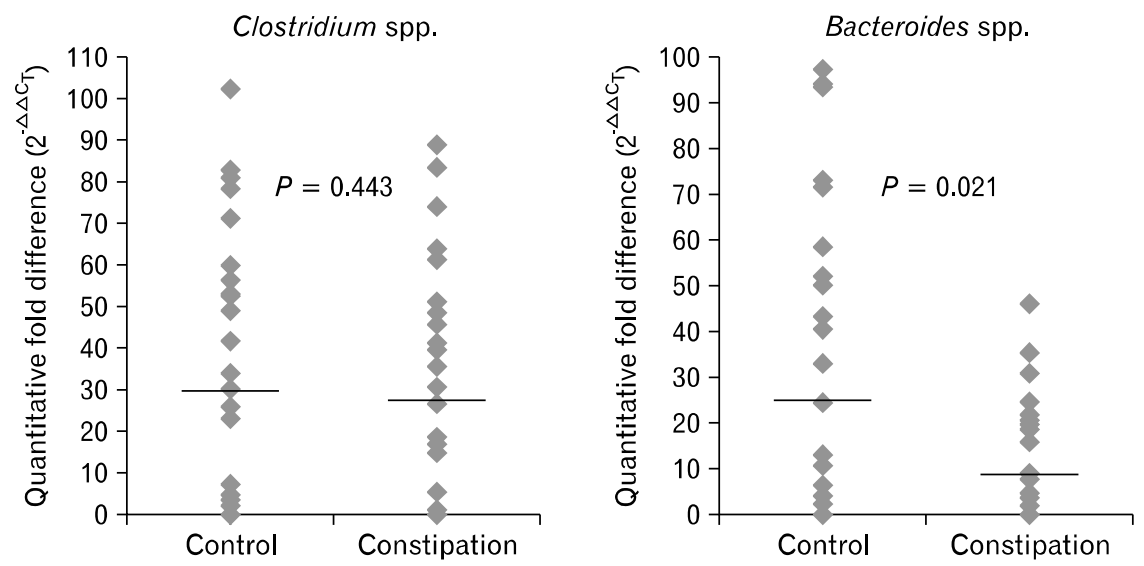

Figure 1. Fold differences, $2^{-\Delta \Delta \mathrm{C}_{\mathrm{T}}}$ was calculated using the comparative $\mathrm{C}_{\mathrm{T}}$ (threshold cycle) method. The fold differences of Bifidobacterium and Bacteroides species in fecal specimens from constipated patients were significantly lower compared to controls $(P=0.030$ and $P=0.021$, respectively; Mann-Whitney test). Bold line shows the median value of each group. 
ferences of Lactobacillus, Bifidobacterium, and Clostridium species between fecal samples from FC patients and controls (Fig. 1).

\section{Comparison of fold differences in concentrations of gut flora after VSL\#3 ingestion}

In controls, fold differences of Lactobacillus, Bifidobacterium, and Bacteroides species increased after ingesting VSL\#3, compared to before ingestion $(P=0.022, P=0.018$, and $P=$ 0.076; Fig. 2). However, in constipated patients, the fold differences of all bacteria did not increase significantly (Fig. 3).

When the fluctuation of gut flora per person before and after ingesting VSL\#3 were analyzed, the mean delta values of fold differences of beneficial bacteria were not significantly different between FC patients and controls $(P=0.228$ for Lactobacillus, $P$ $=0.732$ for Bifidobacterium, and $P=0.448$ for Bacteroides).

\section{Symptomatic Relief of Functional Constipation After Ingesting VSL\#3}

\section{Complete spontaneous bowel movements}

The mean CSBM score per week in constipated patients increased significantly after VSL\#3 ingestion for 2 weeks (6.3 \pm 3.1 week), compared to before ingestion $(2.5 \pm 1.3 /$ week, $P<$ 0.001). Significant improvement of the mean CSBM scores was shown in constipated patients after VSL\# 3 ingestion for 1 week (2.5 \pm 1.3/week vs. $5.4 \pm 2.8$ /week, $P<0.001$; Fig. 4). Additionally, the mean CSBM scores in constipated patients increased significantly after 2 weeks of VSL\# 3 ingestion compared to after 1 week of ingestion $(P=0.032)$.

In healthy controls, no significant difference between the mean CSBM scores before $(6.6 \pm 2.4$ week $)$ and after $(7.6 \pm$
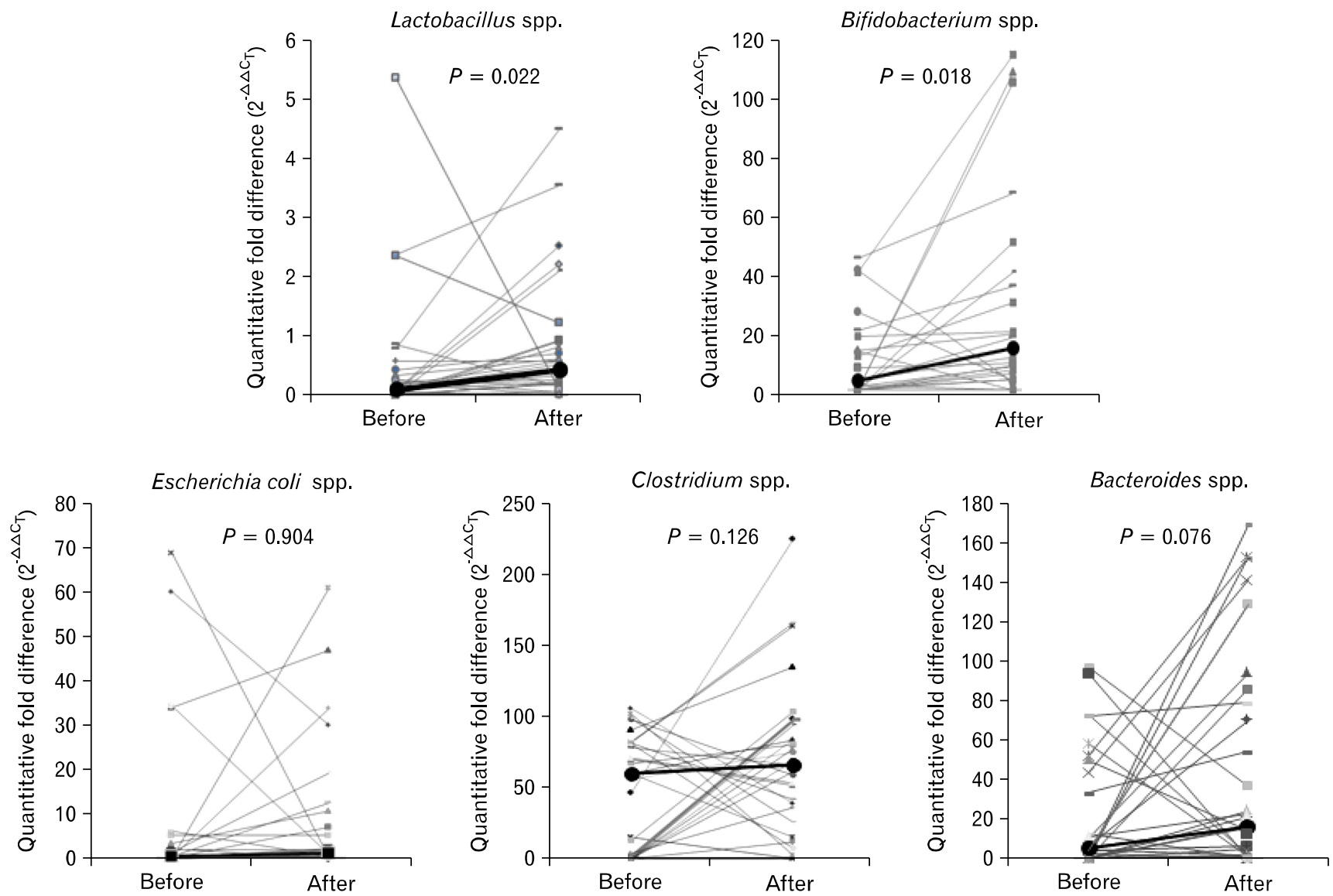

Figure 2. Fold differences in each bacterial gene expression were calculated using the formula $2^{-\Delta \Delta \mathrm{CT}}$ and represented as relative expression after normalization to pre-treated group. In healthy controls, fold differences of Lactobacillus, Bifidobacterium and Bacteroides species increased after VSL\# 3 ingestion compared to before ingestion $(P=0.022, P=0.018$, and $P=0.076$, respectively; Wilcoxon signed-rank test). Bold line shows the median value of each group. 

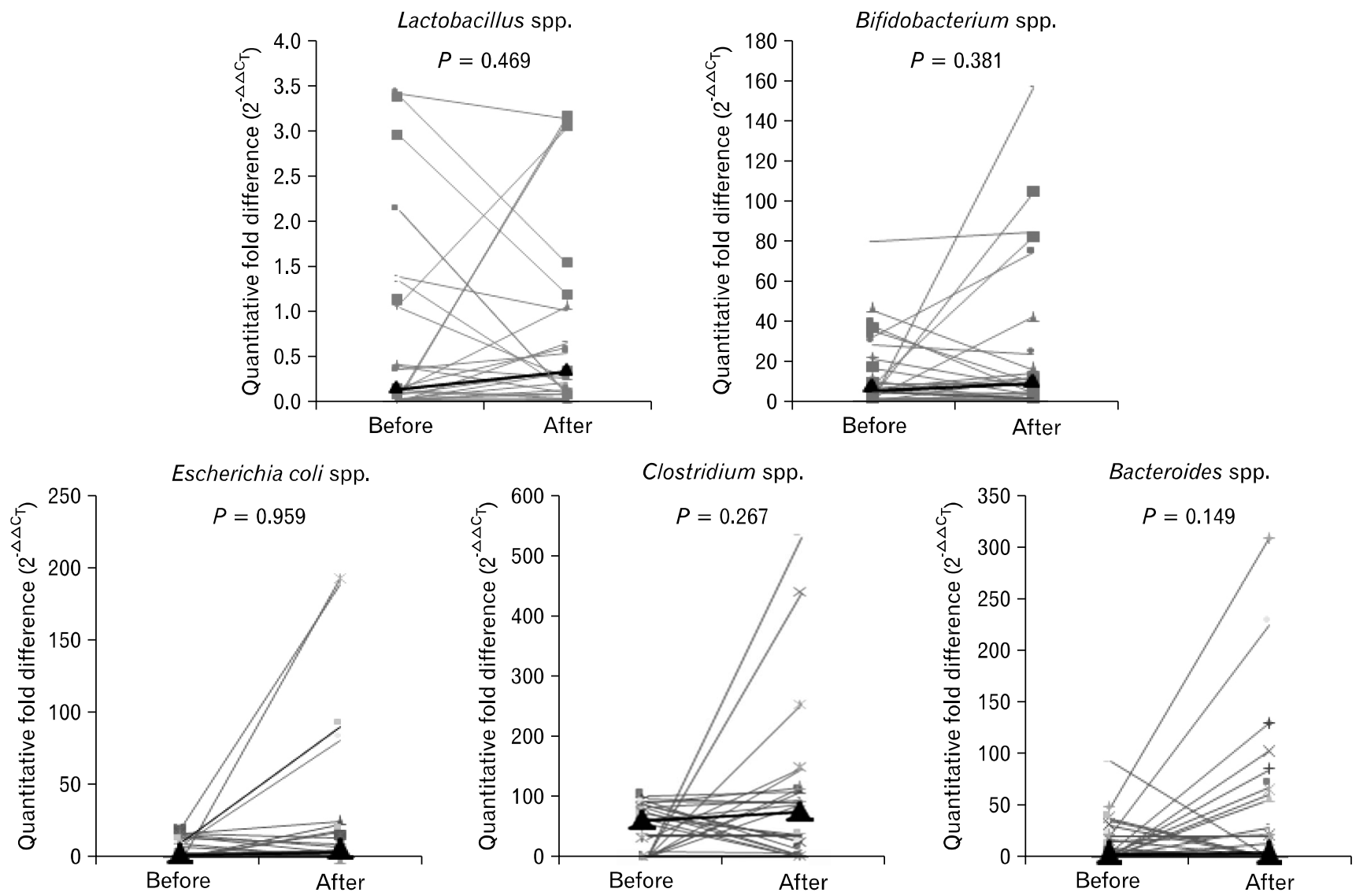

Figure 3. In constipated patients, the fold changes of all flora bacteria did not increase siginificantly (Wilcoxon signed-rank test). Bold line shows the median value of each group.

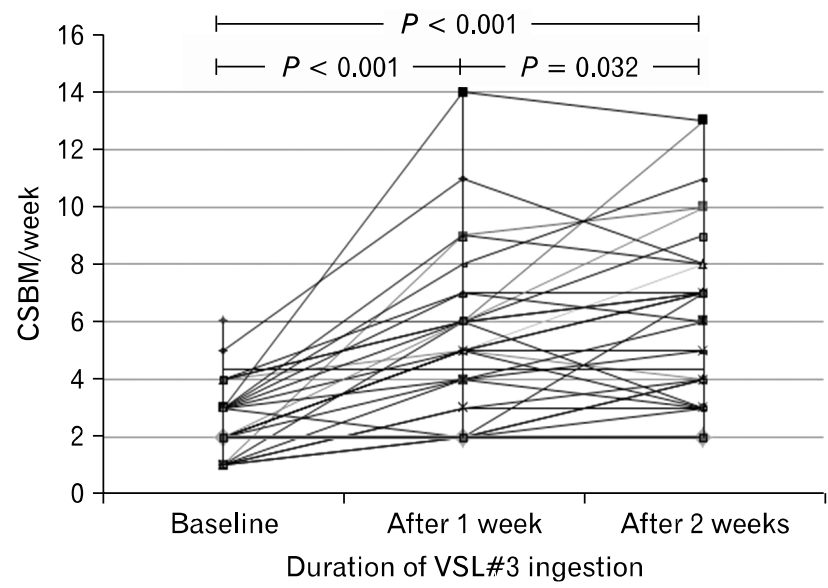

Figure 4. The mean complete spontaneous bowel movement (CSBM) scores per week in constipated patients increased significantly after VSL\# 3 ingestion for 2 weeks ( $6.3 \pm 3.1$ /week), as compared to before ingestion $(2.5 \pm 1.3 /$ week, $P<0.001)$. A significant improvement in the mean CSBM scores was shown in constipated patients after VSL\#3 ingestion for 1 week ( $2.5 \pm 1.3$ /week vs. $5.4 \pm 2.8$ /week, $P<0.001)$. Additionally, the mean CSBM score in constipated patients increased significantly after 2 weeks of VSL\# 3 ingestion as compared to after 1 week of ingestion $(P=0.032$, paired $t$ test). 2.3/week) VSL\#3 ingestion was observed ( $P=0.084$; data not shown).

\section{Stool consistency}

The mean Bristol scores in constipated patients were significantly higher after VSL\# 3 ingestion for 2 weeks $(4.1 \pm 0.9)$ than after ingestion for 1 week $(3.7 \pm 0.9, P=0.014)$, as well as those before VSL\#3 ingestion $(2.6 \pm 0.7, P<0.01)$. A significant improvement in the mean Bristol score of patients was also evident after VSL\#3 ingestion for 1 week ( $P<0.001$; Fig. 5).

In healthy controls, no significant difference in the mean Bristol scores before $(4.0 \pm 0.7)$ and after $(4.3 \pm 0.8, P=$ $0.073)$ VSL\# 3 ingestion was observed.

\section{Subjective symptoms}

Relief of subjective CSBM frequency, stool consistency, and abdominal bloating in the FC group after ingesting VSL\#3 for 2 weeks were reported in $70 \%, 60 \%$, and $47 \%$ of patients, respectively. 


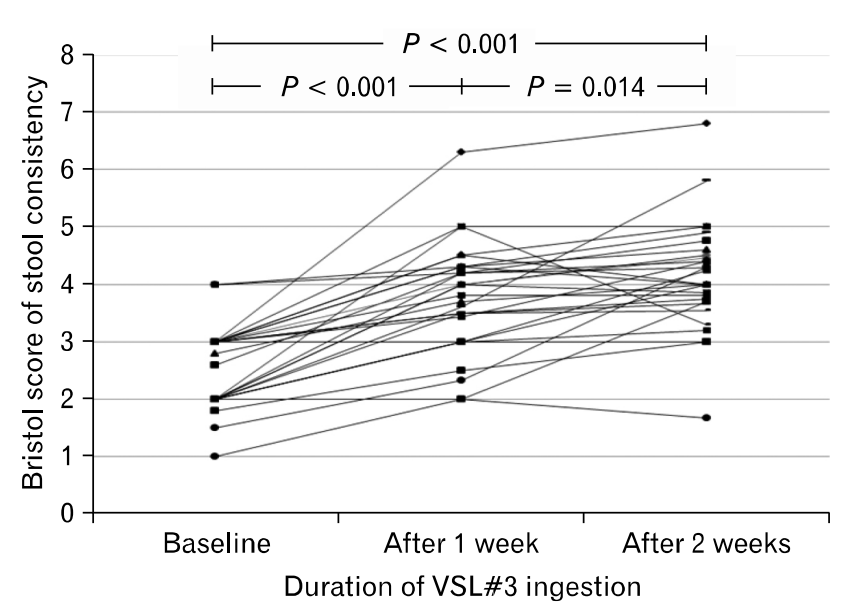

Figure 5. The mean Bristol scores in constipated patients were significantly higher after VSL\# 3 ingestion for 2 weeks than after 1 week (3.7 \pm 0.9 vs. $4.1 \pm 0.9, P=0.014$ ), or before ingestion ( $2.6 \pm 0.7$ to $4.1 \pm 0.9, P<0.001)$. A significant improvement in the mean Bristol scores in constipated patients was also observed after VSL\# 3 ingestion for 1 week $(P<0.001$, paired $t$ test $)$.

After VSL\#3 ingestion, 18 constipated patients who showed symptom improvement were followed up once per month by telephone interview. Constipation-related symptoms including hard stool or reduced defecation frequency, recurred in 11 patients (61\%). Specifically, 9 patients $(50 \%)$ complained of having the original symptom severity: the original hard stool level in 5 patients $(28 \%)$ and reduced defecation frequency in $9(44 \%)$. Of 11 patients with recurrence, $8(72.7 \%)$ reported recurrence of constipation-related symptoms within 1 month (Fig. 6).

\section{Discussion}

The rationale behind research on gut flora in constipated patients is related to the possibility of altering the delayed transit. Our study are meaningful not only because they revealed the quantitative difference in the main gut flora of patients with FC, but also because they show the effect of probiotics on $\mathrm{FC}$, which is likely mediated by the gut flora. Moreover, this is the first study of the effectiveness of VSL\#3 for FC.

There have been limited studies suggesting the effect of a change in gut flora on FC. A previous study showed that Bifidobacterium and Lactobacillus concentration were lower in constipated patients, ${ }^{8}$ in agreement with several older pediatric studies. $^{6,18}$

The role that gut flora play in the pathogenesis of FC remains unclear. A microbiological study using traditional culture

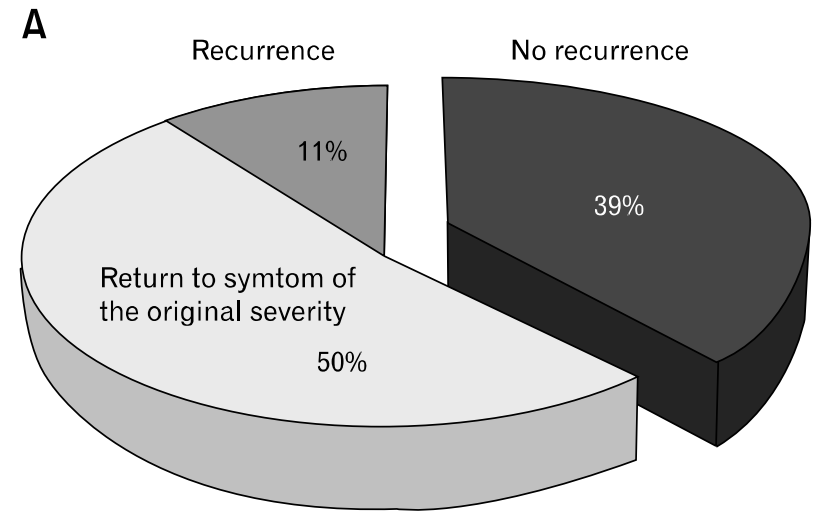

\section{B}

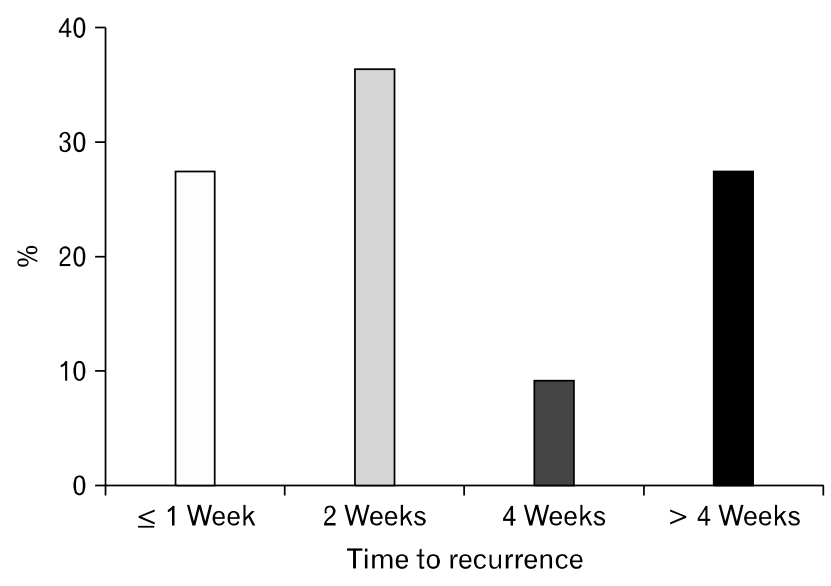

Figure 6. After the VSL\# 3 ingestion period ended, 18 constipated patients who had symptom improvement were followed up monthly by telephone interview. Constipation-related symptoms such as hard stool or reduced defecation frequency recurred in 11 patients $(61 \%)$. In particular, nine patients $(50 \%)$ complained of the original symptom severity (A). Of the 11 patients with recurrence, 8 (72.7\%) reported constipation-related symptoms within 1 month after stopping probiotic ingestion (B).

methods showed that some obligate bacteria, including Lactobacillus, Bifidobacterium, and Bacteroides spp. decreased, while potentially pathogenic bacteria increased, in patients with chronic constipation. The study reported that relief of constipation tended to normalize these findings, suggesting that the gut flora is secondary to, rather than a cause of, constipation. ${ }^{8}$ However, the post-treatment changes in flora were not prominent and were considered to have been analyzed only roughly. The composition of the gut microbiota can change temporarily following bisacodyl treatment.

Several investigators have suggested that changes in the gut flora could alter the motor and secretory functions of the bowel. 
Probiotic studies in both animals and humans showed similar results in terms of stimulating motility, although the direct effect of probiotics on the gut flora has not been investigated. $4,5,12,19,20$

Our results also revealed a change in the gut flora in patients with FC. The fold differences in the concentrations of Bifidobacterium and Bacteroides spp. were significantly lower in the feces of patients with FC compared with in the controls. However, this result does not necessarily mean that Bifidobacterium and Bacteroides decrease colonic transit time. Each strain must be tested on pateints with FC, followed by measurements of colonic transit time. While some studies show that Bifidobacterium spp. decreases gut transit time in healthy adults, there are no studies of the influence of Bacteroides on gut transit. ${ }^{21-23}$

In our study, the VSL\#3 probiotic was administered with the expectation that it would change the gut flora, and improve bowel movement frequency, stool consistency, and bloating in patients. However, no significant changes in the fold differences of levels of the five bacterial taxa after ingesting VSL\#3 were identified in the feces of patients with FC, although gut colonization by lactic acid bacteria in VSL\#3 was confirmed in controls. The mean delta values of fold differences of beneficial bacteria were still not significantly different between FC patients and controls. It could be associated with the data showing that the changes of fold differences before and after treatment were not significant in FC but significant in control, although the fold differences in the concentrations of the beneficial bacteria in FC patients were significantly lower than in controls before ingesting VSL\#3.

The possible reasons for the discrepancy between symptom improvement and gut floral changes after ingesting VSL\#3 include that other bacterial species whose concentrations may have changed in constipated patients were not measured in this study, although these 5 bacterial taxa account for a substantial portion of the fecal flora. The other possibility is that physiologically active substances produced by the gut flora may have affected colon transit, regardless of the fold differences in the concentration of bacterial species. ${ }^{12,24-26}$ Several studies have demonstrated that administering probiotics or prebiotics such as Lactobacillus-enriched artichoke reduced visceral hypersensitivity and inflammation, or relieved symptoms of constipation, without altering bacterial populations. ${ }^{26-28}$ Probiotics are capable of lowering intracolonic $\mathrm{pH}$ due to bacterial SCFA production. In turn, a lower $\mathrm{pH}$ enhances colonic peristalsis, decreasing transit time. ${ }^{29}$ It is not necessary for probiotics to colonize the human intestine to exert a beneficial effect on health. ${ }^{1}$
Bacterial colonization by short-term ingestion of probiotics may not be effective in a diseased bowel. The constipated subjects enrolled in this study had a long symptom history, and the laxatives previously used were not effective in relieving symptoms. Our study revealed that a 2 -week ingestion of VSL\# 3 resulted in colonization of the gut in controls. Although CSBMs and stool consistency tended to increase, no clinically significant difference in bowel parameters was observed in controls. Further studies are needed to confirm the difference in colonization between controls and patients with $\mathrm{FC}$ after probiotic treatment, and to identify the underlying mechanism.

Much clinical experience and some data suggest that probiotics may be beneficial in many types of intestinal disorders, especially diarrheal diseases. ${ }^{30}$ Orally administered probiotics have been considered to target the motor and neural apparatus in post-infective gut dysfunctions. ${ }^{25}$ Probiotics including lactic acid bacteria were shown to be effective for the relief of abdominal bloating in patients with diarrhea-predominant irritable bowel syndrome (D-IBS), but did not show a significant alteration in gut transit. ${ }^{31,32}$ Studies involving the administration of a probiotic, B. lactis DN-173 010, showed improved colonic transit times in both a healthy population and constipated patients. ${ }^{20,33,34}$ The mechanism of action of the intestinal flora in this regard remains unclear. The data published to date do not provide sufficient evidence to support a general recommendation of the use of probiotics for the treatment of $\mathrm{FC}^{29}$

The VSL\#3 probiotic was chosen because studies have suggested that in constipated patients, probiotics may be more effective if multiple strains are administered together. ${ }^{12,29,35}$ VSL\#3 contains eight strains of live lactic acid bacteria that were selected to produce an optimal synergistic effect. In several D-IBS studies, lactic acid bacteria were shown to reduce abdominal bloating compared to a placebo and influence gut transit and bowel dysfunction, ${ }^{31,32}$ However, no reports are available on the effectiveness of VSL\#3 for FC.

The duration of probiotic administration generally varies from 2 to 6 weeks or more, at least in D-IBS patients. We administered the probiotic to all subjects for 2 weeks. Administration for longer periods may have influenced patient compliance, and thus a shorter treatment periods was used. Instead of using a 2-week study period, the daily dose was doubled. None of the patients complained of side effects, and all showed good compliance with treatment.

The fact that $70 \%$ of the patients were satisfied with their symptomatic relief of bowel movement frequency is considered to 
be a very good outcome of VSL\#3 treatment. However, it should be noted that at least $30 \%$ of constipated patients were unsatisfied with the double-dose VSL\#3 treatment. In addition, $61 \%$ of patients who showed improvement had their constipation symptoms recur after treatment cessation, mostly within 1 month. Although the gut flora composition of an individual can fluctuate under different circumstances, such as dietary interventions, acute infection, and antibiotic treatment, it usually returns to normal or remains constant. ${ }^{1}$ According to our results, some constipated patients may derive substantial benefit from a combination of multiple probiotic strains. However, the treatment may need to be administered on a cyclical schedule or repeatedly because the resultant favorable effect is temporary. ${ }^{36}$

Quantitative analysis of fecal bacteria shows important differences in yield that are not always detectable by conventional culture techniques. ${ }^{1}$ The molecular method used in our study, however, had limitations in terms of estimating the composition of the colonic flora and providing a generalized view of the diverse intestinal flora compared with pyrosequencing methods. We used a comparative CT method to normalize the bacterial gene expression to total $16 \mathrm{~S}$ rRNA in each fecal sample. This compensatory analysis is considered to be resonable to compare gut flora between groups in this study.

We also acknowledge a limitation of not involving a metabolomics study such as short chain fatty acids and $\mathrm{pH}$. Additionally, the effect of VSL\#3 on constipation symptoms was evaluated without a placebo group in this pilot study. However, a symptom assessment was conducted based mainly on CSBMs and stool consistency, which are relatively objective, as well as numerical counts. It is difficult to conclude that only a placebo effect was responsible for the improved symptoms of constipated patients with a long symptom history, and who had not responded to various laxatives.

Nevertheless, this pilot study is useful because it shows that gut flora can impact disturbed bowel function, as evidenced by the improvement of FC after short-term administration of a probiotic, as well the difference in gut flora concentrations between constipated patients and controls.

In conclusion, quantitative alterations of specific bacterial groups in the gut flora were found in patients with FC. VSL\#3 could be effective in terms of improving clinical symptoms in constipated patients, although its direct effect on the gut flora of constipated patients remain unclear. More studies should aim to elucidate the mechanism underlying the effect of short-term treatment with VSL\#3 on FC, particularly in light of its metab- olomic effect on the gut flora.

\section{Acknowledgements}

The authors especially thank Yang Hee Joo of the Ewha Medical Research Institute for her contribution to this investigation. The authors also thank Prof. Kijeong Kim of Chung Ang University College of Medicine and Prof. So Yoen Woo of Ewha Womans University School of Medicine for their advice on the experimental methodology.

\section{References}

1. Guarner F, Malagelada JR. Gut flora in health and disease. Lancet 2003;361:512-519.

2. Simon GL, Gorbach SL. Intestinal flora in health and disease. Gastroenterology 1984;86:174-193.

3. Hooper LV, Wong MH, Thelin A, Hansson L, Falk PG, Gordon JI. Molecular analysis of commensal host-microbial relationships in the intestine. Science 2001;291:881-884.

4. Husebye E, Hellström PM, Midtvedt T. Intestinal microflora stimulates myoelectric activity of rat small intestine by promoting cyclic initiation and aboral propagation of migrating myoelectric complex. Dig Dis Sci 1994;39:946-956.

5. Caenepeel P, Janssens J, Vantrappen G, Eyssen H, Coremans G. Interdigestive myoelectric complex in germ-free rats. Dig Dis Sci 1989;34:1180-1184.

6. Zoppi G, Cinquetti M, Luciano A, Benini A, Muner A, Bertazzoni Minelli E. The intestinal ecosystem in chronic functional constipation. Acta Paediatr 1998;87:836-841.

7. Drossman DA, Li Z, Andruzzi E, et al. U.S. householder survey of functional gastrointestinal disorders. Prevalence, sociodemography, and health impact. Dig Dis Sci 1993;38:1569-1580.

8. Khalif IL, Quigley EM, Konovitch EA, Maximova ID. Alterations in the colonic flora and intestinal permeability and evidence of immune activation in chronic constipation. Dig Liver Dis 2005;37: 838-849.

9. Chassard C, Dapoigny M, Scott KP, et al. Functional dysbiosis within the gut microbiota of patients with constipated-irritable bowel syndrome. Aliment Pharmacol Ther 2012;35:828-838.

10. Suau A, Bonnet R, Sutren M, et al. Direct analysis of genes encoding 16S rRNA from complex communities reveals many novel molecular species within the human gut. Appl Environ Microbiol 1999;65: 4799-4807.

11. Malinen E, Rinttilä T, Kajander K, et al. Analysis of the fecal microbiota of irritable bowel syndrome patients and healthy controls with real-time PCR. Am J Gastroenterol 2005;100:373-382.

12. Ouwehand AC, Lagström H, Suomalainen T, Salminen S. Effect of probiotics on constipation, fecal azoreductase activity and fecal mucin content in the elderly. Ann Nutr Metab 2002;46:159-162.

13. Koebnick C, Wagner I, Leitzmann P, Stern U, Zunft HJ. Probiotic beverage containing Lactobacillus casei Shirota improves gastrointestinal symptoms in patients with chronic constipation. Can J 
Gastroenterol 2003;17:655-659.

14. Yang $\mathrm{YX}, \mathrm{He} \mathrm{M}, \mathrm{Hu} \mathrm{G}$, et al. Effect of a fermented milk containing Bifidobacterium lactis DN-173010 on Chinese constipated women. World J Gastroenterol 2008;14:6237-6243.

15. Heaton KW, Ghosh S, Braddon FE. How bad are the symptoms and bowel dysfunction of patients with the irritable bowel syndrome? A prospective, controlled study with emphasis on stool form. Gut 1991;32:73-79.

16. Carroll IM, Chang YH, Park J, Sartor RB, Ringel Y. Luminal and mucosal-associated intestinal microbiota in patients with diarrhea-predominant irritable bowel syndrome. Gut Pathog 2010;2:19.

17. Schmittgen TD, Livak KJ. Analyzing real-time PCR data by the comparative C(T) method. Nat Protoc 2008;3:1101-1108.

18. Kirgizov IV, Sukhorukov AM, Dudarev VA, Istomin AA. Hemostasis in children with dysbacteriosis in chronic constipation. Clin Appl Thromb Hemost 2001;7:335-338.

19. Nakamura T, Nishida S, Mizutani M, Iino H. Effects of yogurt supplemented with brewer's yeast cell wall on constipation and intestinal microflora in rats. J Nutr Sci Vitaminol 2001;47:367-372.

20. Riezzo G, Orlando A, D'Attoma B, et al. Randomised clinical trial: efficacy of Lactobacillus paracasei-enriched artichokes in the treatment of patients with functional constipation - a double-blind, controlled, crossover study. Aliment Pharmacol Ther 2012;35:441-450.

21. Wexler HM. Bacteroides: the good, the bad, and the nitty-gritty. Clin Microbiol Rev 2007;20:593-621.

22. Marteau $\mathrm{P}$, Cuillerier $\mathrm{E}$, Meance $\mathrm{S}$, et al. Bifidobacterium animalis strain DN-173 010 shortens the colonic transit time in healthy women: a double-blind, randomized, controlled study. Aliment Pharmacol Ther 2002;16:587-593.

23. Waller PA, Gopal PK, Leyer GJ, et al. Dose-response effect of Bifidobacterium lactis $\mathrm{HN} 019$ on whole gut transit time and functional gastrointestinal symptoms in adults. Scand J Gastroenterol 2011;46: 1057-1064.

24. Borriello SP. Bacteria and gastrointestinal secretion and motility. Scand J Gastroenterol Suppl 1984;93:115-121.

25. Verdú EF, Bercik P, Collins SM. Effect of probiotics on gastrointestinal function: evidence from animal models. Therap Adv
Gastroenterol 2009;2:31-35.

26. Valerio F, Russo F, de Candia S, et al. Effects of probiotic Lactobacillus paracasei-enriched artichokes on constipated patients: a pilot study. J Clin Gastroenterol 2010;44(suppl 1):S49-S53.

27. Verdú EF, Bercik P, Verma-Gandhu M, et al. Specific probiotic therapy attenuates antibiotic induced visceral hypersensitivity in mice. Gut 2006;5 5:182-190.

28. Kleessen B, Sykura B, Zunft HJ, Blaut M. Effects of inulin and lactose on fecal microflora, microbial activity, and bowel habit in elderly constipated persons. Am J Clin Nutr 1997;65:1397-1402.

29. Chmielewska A, Szajewska H. Systematic review of randomised controlled trials: probiotics for functional constipation. World J Gastroenterol 2010;16:69-75.

30. Rolfe RD. The role of probiotic cultures in the control of gastrointestinal health. J Nutr 2000;130:396S-402S.

31. Kim HJ, Camilleri M, McKinzie S, et al. A randomized controlled trial of a probiotic, VSL\#3, on gut transit and symptoms in diarrhoea-predominant irritable bowel syndrome. Aliment Pharmacol Ther 2003;17:895-904.

32. Nobaek S, Johansson ML, Molin G, Ahrné S, Jeppsson B. Alteration of intestinal microflora is associated with reduction in abdominal bloating and pain in patients with irritable bowel syndrome. Am J Gastroenterol 2000;95:1231-1238.

33. Agrawal A, Houghton LA, Morris J, et al. Clinical trial: the effects of a fermented milk product containing Bifidobacterium lactis DN-173 010 on abdominal distension and gastrointestinal transit in irritable bowel syndrome with constipation. Aliment Pharmacol Ther 2009; 29:104-114.

34. Picard C, Fioramonti J, Francois A, Robinson T, Neant F, Matuchansky C. Review article: bifidobacteria as probiotic agents - physiological effects and clinical benefits. Aliment Pharmacol Ther 2005; 22:495-512.

35. Bekkali NL, Bongers ME, Van den Berg MM, Liem O, Benninga MA. The role of a probiotics mixture in the treatment of childhood constipation: a pilot study. Nutr J 2007;6:17.

36. Salminen S, von Wright A, Morelli L, et al. Demonstration of safety of probiotics - a review. Int J Food Microbiol 1998;44:93-106. 